ÉGYPTE monde arabe

\section{Égypte/Monde arabe}

15-16 | 1993

Les crises soudanaises des années 80

\title{
Le renouvellement de l'accord entre l'Égypte et le Fonds monétaire international et ses conséquences
}

\section{Louis Blin}

\section{OpenEdition}

1 Journals

Édition électronique

URL : https://journals.openedition.org/ema/1110

DOI : 10.4000/ema. 1110

ISSN : 2090-7273

Éditeur

CEDEJ - Centre d'études et de documentation économiques juridiques et sociales

Édition imprimée

Date de publication : 31 décembre 1993

Pagination : 297-321

ISSN : 1110-5097

\section{Référence électronique}

Louis Blin, « Le renouvellement de l'accord entre l'Égypte et le Fonds monétaire international et ses conséquences », Égypte/Monde arabe [En ligne], 15-16 | 1993, mis en ligne le 08 juillet 2008, consulté le 07 juillet 2022. URL : http://journals.openedition.org/ema/1110; DOI : https://doi.org/10.4000/ema. 1110

Ce document a été généré automatiquement le 7 juillet 2022.

Tous droits réservés 


\title{
Le renouvellement de l'accord entre l'Égypte et le Fonds monétaire international et ses conséquences
}

\author{
Louis Blin
}

1 Le conseil d'administration du Fonds monétaire international a ratifié, au cours d'une séance ayant duré trois heures le 20 septembre 1993, l'accord de principe conclu le 22 juillet précédent au Caire avec le gouvernement égyptien. Sa décision permet à l'Égypte de bénéficier d'une "facilité de financement élargie» (Extended Fund Facility de 400 millions de droits de tirages spéciaux (DTS, 569 millions \$), qui représente $59 \%$ de sa quote-part au Fonds (678,4 millions DTS) et qu'elle pourra prélever par tranches semestrielles jusqu'au 20 septembre 1996, date de son échéance. L'Égypte s'engage ainsi dans la deuxième phase de l'ajustement structurel de son économie, selon des modalités que nous exposerons, après avoir détaillé le déroulement des négociations ayant conduit à l'accord et avant d'en analyser les conséquences.

Le déroulement des négociations

2 La conclusion d'un accord entre l'Égypte et le FMI donne toujours lieu à des tractations serrées. Le nouvel accord, qui ne constitue pourtant qu'un prolongement des réformes mises en chantier depuis 1991 et qui suit la procédure normale des interventions du Fonds, n'a pas échappé à la règle.

Le résultat de négociations difficiles

3 Le nouvel accord a été conclu à l'issue de près d'un an de négociations quasipermanentes, les délégations du FMI et de la Banque mondiale s'étant longtemps succédé sans succès au Caire. Il aurait dû entrer en vigueur immédiatement après la date prévue pour l'achèvement de l'accord de confirmation ${ }^{1}$, le 30 novembre 1992, si les autorités égyptiennes avaient respecté le calendrier de mise en œuvre des réformes prévues dans son cadre.

4 Elles l'ont fait dans un premier temps, puisque le conseil d'administration du FMI donnait le 13 décembre 1991 - approximativement dans les délais convenus - son agrément à la poursuite de cet accord, qui prévoyait une évaluation semestrielle, par 
des missions du FMI, des mesures mises en œuvre par le gouvernement. La seconde évaluation devait en conséquence être achevée le 15 Juin 1992, mais la politique fiscale et d'ajustement ayant divergé par rapport aux objectifs tracés, les autorités égyptiennes ont demandé un délai pour sa misé en œuvre. Le conseil d'administration du Fonds a accepté leur requête le 30 novembre suivant et accordé un report de l'échéance de l'accord de confirmation au 1er mars $1993^{2}$. Le 9 janvier, le gouvernement a demandé un deuxième prolongement de l'accord jusqu'au 31 mai 1993, souhait auquel le conseil d'administration du Fonds a accédé le 26 janvier, après avoir approuvé les conclusions de la deuxième évaluation de l'accord par ses experts. L'Égypte a également demandé la substitution à l'accord de confirmation d'un accord de plus longue durée. Suite à l'agrément du FMI, la Banque mondiale a ratifié, le 12 mars 1993. le déblocage de la deuxième tranche de $50 \%$ du prêt d'ajustement structurel de 300 millions \$ signé le 22 novembre 1991, qui accompagnait le programme du Fonds.

5 L'examen de la mise en œuvre des réformes économiques depuis la conclusion de l'accord de confirmation montre qu'après avoir pris conscience des excellents résultats obtenus en 1991, qui dépassaient même les attentes des institutions financières internationales, les autorités égyptiennes semblent avoir considéré, durant toute l'année 1992, que ces performances leur permettaient de marquer une pause dans les réformes. Hormis la nouvelle loi foncière, les lois votées par le Parlement (sur les banques, le marché financier) n'ont en effet pas été mises en vigueur faute de décrets d'application, et nombre de mesures ont été reportées. Le représentant résident du FMI au Caire regrettait, en juin 1992, cet état de lait en ces termes : « Une décision politique a été prise de progresser à un certain rythme. $»^{3}$

6 Le gouvernement égyptien n'était alors guère sensible à l'argumentation du FMI selon laquelle la situation financière extérieure exceptionnelle de l'Égypte ne devait pas servir de prétexte pour différer des réformes structurelles douloureuses, mais représentait au contraire une opportunité unique de les mettre en œuvre dans un contexte favorable. L'une des principales difficultés auxquelles se heurtent les programmes d'ajustement structurel est en effet que les gouvernements - y compris celui de l'Égypte - ne se résolvent habituellement à les appliquer que pour répondre à une crise de leurs paiements extérieurs, alors que leur mise en œuvre nécessite des crédits importants. C'est pourquoi ces programmes sont accompagnés de prêts du FMI, de la Banque mondiale et parfois d'autres organismes. L'énorme excédent du compte courant égyptien apparu à compter de 1991 diminuait l'importance de la réduction de la dette extérieure, enjeu de la poursuite du programme. Le gouvernement se contentait d'adopter, à partir de décembre 1992, certaines mesures (fiscales, monétaires et lancement d'appels d'offres pour des privatisations) propres à ménager les institutions financières internationales, avec lesquelles étaient habilement négociés les délais de grâce mentionnés, en s'appuyant sur les difficultés imprévues causées par les attentats islamistes apparus l'été précédent et le tremblement de terre du Caire du 12 octobre 1992.

7 Ce n'est cependant qu'après s'être assuré des intentions de la nouvelle administration américaine à l'égard de l'Égypte en se rendant personnellement le 6 avril 1993 à Washington, où il s'entretenait avec le directeur général du FMI, puis avec son homologue américain, que le président Moubarak décidait de donner une nouvelle impulsion aux réformes, tout en négociant les conditions les plus douées possibles pour leur application. Le premier signe d'une reprise en main de cette dérive vers 
l'immobilisme était donné le 18 avril suivant par la promotion du haut fonctionnaire en charge du dossier des négociations avec le FMI au poste de ministre d'État ${ }^{4}$ auprès de la présidence $\mathrm{du}$ Conseil. On ne pouvait tenir pour un hasard que ce responsable, M. Youssef Boutros-Ghali, soit un ancien employé du Fonds et le neveu de M. Boutros Boutros-Ghali, secrétaire général d'une ONU récemment réconciliée avec l'administration américaine.

8 Ce n'est qu'après l'expiration du délai de grâce accordé par le Fonds et suite à d'inhabituelles critiques publiques exprimées par la Banque mondiale, que le président égyptien réunissait, le 14 juin, le Premier ministre et les ministres économiques pour leur donner les instructions nécessaires à une conclusion rapide. M. Youssef BoutrosGhali pouvait alors négocier, fort du soutien américain. Bien que les tractations se soient encore poursuivies plus d'un mois, les autorités égyptiennes ont souscrit aux desiderata du Fonds. Afin de s'assurer que son conseil d'administration ratifie l'accord de principe conclu au niveau des experts en juillet, elles ont commencé à appliquer, dès la fin de Juillet, les engagements contenus dans leur nouvelle "lettre d'intention", dont la mouture définitive était envoyée le 18 août suivant au FMI. Ces mesures délicates ont ainsi été prises après la clôture de la session parlementaire, ce qui évitait certains débats au sein d'un Parlement réputé dirigiste. Le 20 septembre enfin, le conseil d'administration du Fonds a accepté les termes de la lettre d'intention, renouvelant par là-même l'accord de 1991, sans toutefois que le gouvernement égyptien ne signe formellement de document commun avec le FMI. C'est pourquoi le terme de, «nouvel accord » entre l'Égypte et le Fonds est abusif stricto sensu, quoiqu'il corresponde bien à la réalité. Nous l'emploierons donc, bien que le Premier ministre, répondant aux détracteurs traditionnels des " diktats du FMI ", ait affirmé avec raison que le gouvernement n'avait signé aucun accord avec celui-ci ${ }^{5}$.

9 Le FMI faisant du respect du programme arrêté avec la Banque mondiale, concentré sur l'ajustement structurel, une des conditions de la poursuite de ses relations avec l'Égypte, cette dernière devra également conclure un nouvel accord avec la Banque. Vu les taux élevés des prêts de la Banque mondiale, l'Égypte n'a pourtant tiré, en juin 1993, que $67 \%$ de l'ensemble des sommes que cette dernière lui avait attribuées. Le nouveau programme, mis au point à l'occasion d'une mission de la Banque en Égypte achevée le 4 septembre, ne prévoit donc pas de deuxième prêt à l'ajustement structurel. Il porte sur la poursuite du plan d'ajustement défini en 1991, suivant les engagements pris à l'égard du FMI.

Un accord conclu avec dix mois de retard

Le nouvel accord avec le FMI succède, avec dix mois de retard sur l'échéance prévue, à l'accord de confirmation (stand by) signé le 17 mai 1991, qui autorisait l'Égypte à emprunter au Fonds 278 millions DTS pendant 18 mois. Sa conclusion équivaut à l'attribution d'un satisfecit du Fonds à l'Égypte quant à la mise en œuvre de l'accord précédent. D'une durée double de celui-ci, le nouvel accord prévoit une poursuite de la politique de stabilisation entreprise par l'Égypte avec succès et l'approfondissement de l'ajustement structurel à moyen terme. Les mesures adoptées dans son cadre seront évaluées tous les six mois par une mission du FMI. L'Égypte, dont les réserves en devises dépassent 14 milliards $\$$, a indiqué au Fonds qu'elle n'avait pour le moment pas l'intention de procéder aux tirages autorisés par le nouvel accord. Au début de 1993, le gouvernement avait déjà averti le FMI qu'il n'utiliserait pas son droit de tirer la dernière tranche de 130,8 millions DTS à laquelle il pouvait prétendre au titre de 
l'accord de confirmation, évitant ainsi de payer l'intérêt de 7 \% dont elle était assortie, alors que ses réserves en devises sont rémunérées au taux d'environ $3 \%$ seulement.

11 L'intérêt du nouvel accord pour l'Égypte ne réside donc pas dans les crédits dont il est assorti, mais dans la réduction de sa dette extérieure d'une deuxième tranche de $15 \%$ dont il était la condition, aux termes des dispositions édictées par le Club de Paris le 25 mai $1991^{6}$. Vu le retard mis à renouveler l'accord, la dernière réduction de $20 \%$ de la dette prévue par ces mêmes dispositions ne devrait intervenir qu'à la fin de 1994, sous réserve de l'agrément du FMI, alors qu'elle était initialement envisagée pour juillet 1994. Une délégation égyptienne s'est entretenue en juillet 1993 à Paris avec le secrétariat du Club de Paris pour examiner les modalités de cette nouvelle réduction de dette, qui porte sur 2,46 milliards \$, somme équivalente à la première remise obtenue en 19917. M. Kamal el-Ganzouri, vice-premier ministre et ministre du Plan, a par ailleurs évalué, le 22 septembre, les besoins de l'Égypte en crédits extérieurs à 12 milliards \$ au cours des trois années couvertes par l'accord. Une réunion du groupe consultatif des bailleurs de fonds de l'Égypte est prévue en janvier 1994 pour examiner cette demande. Le groupe consultatif réuni en juillet 1991 au lendemain de l'accord précédent avec le, FMI s'était engagé à attribuer 8 milliards \$ d'aide à l'Égypte pendant une période de deux ans 8 .

Les nouveaux engagements du gouvernement égyptien

12 La lettre d'intention remise au FMI par le gouvernement égyptien couvre la période allant du 1er juillet 1993 au 30 juin 1996. Elle détaille de manière précise ses engagements de politique économique jusqu'à la mi-1995, est plus vague pour l'année suivante et inclut les réformes mises en œuvre en juillet 1993 (exposées dans cette revue dans notre chronique économique du troisième trimestre 1993). Adoptées avant l'acceptation de la lettre d'intention par le FMI, ces réformes en constituaient un préalable et peuvent donc plutôt être considérées comme les mesures terminales prises dans le cadre de l'accord précédent, qui ont permis au Fonds de le considérer comme mené à bon terme. La deuxième phase de l'ajustement structurel couverte par le nouvel accord ne se distingue de la première que par une réforme plus profonde de l'économie. Elle ne fait que continuer le travail entrepris dans chaque secteur, ce qui permet à notre exposé de suivre le plan déjà adopté pour l'analyse de la première phase de l'ajustement structurel ${ }^{9}$, facilitant ainsi les comparaisons. Les nouvelles mesures touchent principalement les domaines suivants : fiscalité, politique monétaire, secteur financier, restructuration du secteur public et privatisations, promotion du secteur privé, politique tarifaire et commerce extérieur.

Réforme fiscale

La politique fiscale visera à compenser la baisse des droits de douane par une hausse des recettes fiscales intérieures. Elle prévoit l'instauration de la TVA et la réforme des impôts sur le revenu et sur les sociétés.

Dans une première étape, la taxe sur les ventes a été modifiée en juillet 1993 (voir chronique économique du troisième trimestre 1993, chapitre « finances »). La deuxième étape, également destinée à préparer l'introduction de la TVA, prévoit la soumission au Parlement, à la fin de 1993, d'un texte étendant cette taxe à de nouveaux services à partir du 1er Janvier 1994. Cette réforme devrait procurer 200 millions LE de revenus additionnels à l'Etat en année pleine. La substitution de la TVA à la taxe sur les ventes sera opérée le 1er juillet 1995 au plus tard. Elle sera étendue à la plupart des services, 
au taux de $10 \%$, ainsi qu'aux matières premières, et prévoit une déductibilité totale sur les consommations intermédiaires et les achats de biens d'équipement.

La réforme de la fiscalité sur les revenus porte sur l'unification des impôts sur le revenu, l'amélioration de leur recouvrement et la réduction du taux maximal d'imposition. Jusqu'à présent, les particuliers doivent s'acquitter d'impôts sur la terre, la propriété, les bénéfices commerciaux et industriels, les salaires ou revenus des professions libérales. S'y ajoute l'impôt qualifié de "général ", qui couvre les autres revenus et possède 22 tranches allant de $9 \%$ d'imposition à partir de 3.000 LE de revenus annuels jusqu'à $65 \%$ au-dessus de 200.000 LE. Le nouvel impôt s'appliquera à partir de 5.000 LE de revenus, ses taux iront de 5 à $50 \%$ et ses barèmes seront plus simples. Appelé impôt unique sur les revenus, il doit entrer en vigueur en 1994 et s'appliquer sur les revenus perçus en 1993. Un projet de loi en ce sens a été déposé au Parlement le 15 juin 1993.

D'autre part, une retenue à la source, au taux de $5 \%$, sera instaurée sur les revenus de créances perçus par les agents non financiers. La fiscalité sur les entreprises sera également modifiée, avec un taux plancher de $1 \%$ sur le chiffre d'affaires. Ces deux impôts devraient procurer des revenus additionnels respectifs de 360 et 600 millions LE au budget de l'Etat en 1993-94.

Politique monétaire

La croissance de la masse monétaire est passée de $14,3 \%$ en $1991-92$ à $16,4 \%$ en 1992-93, principalement en raison de l'accumulation de réserves en devises $(14,8$ milliards \$ à la fin juin 1993), et l'objectif est d'abaisser son expansion à $12 \%$ en 1993-94 afin d'atténuer les tensions inflationnistes. Les crédits aux entreprises devraient progresser de $18 \%$ et leur taux est appelé à décliner lentement, en ligne avec la baisse des taux sur les bons du Trésor, mais en prenant garde aux éventuelles fuites de capitaux. Une nouvelle loi sur les transactions en devises sera soumise au Parlement avant la lin de 1993, qui officialisera la libre convertibilité obtenue de facto par la livre égyptienne et abolira tous les justificatifs pour les rapatriements de devises.

La politique en matière de taux de change visera à garantir à long terme la compétitivité extérieure de l'Égypte, alors que la stabilité de la livre par rapport au dollar de l'été 1991 à l'automne 1992, suivie d'un très léger effritement, a eu pour conséquence son appréciation en termes réels: en raison du différentiel de taux d'inflation, elle s'est appréciée de $8 \%$ en 1991-92 et de $9 \%$ en 1992-93 vis-à-vis du dollar, et davantage encore vis-à-vis des monnaies des autres grands partenaires commerciaux de l'Égypte, qui se sont dépréciées dans le même temps vis-à-vis du dollar. Cette situation va à rencontre de la politique de promotion des exportations poursuivie dans le cadre de la libéralisation de l'économie. Vu l'afflux de devises enregistré depuis 1991 par l'Égypte, la Banque centrale a dû intervenir à maintes reprises pour empêcher la livre de s'apprécier en termes nominaux par rapport à la monnaie américaine. La politique monétaire implicite dans la lettre d'intention est donc de favoriser une légère dépréciation de la livre.

Des seuils trimestriels minima pour les réserves de change ont été fixés jusqu'en juin 1994, ainsi que des plafonds pour l'endettement externe à court terme, nonconcessionnel ou garanti, du gouvernement et des établissements publics (et non des entreprises publiques), les avoirs en livres détenus par la Banque centrale, les emprunts domestiques des entités publiques (hors entreprises relevant de la loi 203) et les réserves en devises. Le gouvernement s'engage enfin à régler le problème de l'accord 
bilatéral de paiement avec le Soudan et à apurer avant octobre 1993 ses arriérés à l'égard de ses créanciers non membres du Club de Paris. L'Égypte a récemment réglé ses derniers arriérés de paiement envers l'Italie, dernier membre du Club de Paris à l'égard duquel il en subsistait. Alors que tous les arriérés devaient avoir disparu dès la fin de 1991, suivant l'accord conclu en juillet précédent avec le Club de Paris, l'Égypte accusait encore, à la mi-1993, 423 millions d'arriérés sur la dette contractée à l'égard des pays non-membres du Club de Paris (Chine, Iran, Brésil, Roumanie et Tchécoslovaquie surtout) et de sources commerciales, contre 755 millions $\$$ un an plus tôt. Le gouvernement avait déjà souscrit, au début de 1993, à une promesse similaire pour la fin du mois de mai suivant.

Réforme du secteur financier

20 La réforme du secteur financier touchera les banques, les compagnies d'assurances, le marché financier, les retraites et la sécurité sociale.

21 Les taux des réserves obligatoires sur les dépôts en devises dans les banques seront ramenés de $15 \%$ à $10 \%$ pour les devises à la fin de 1993. Le taux correspondant de $15 \%$ pour les dépôts en livres, eux non rémunérés, sera lui aussi abaissé à une date postérieure à la mi-1995, mais non encore fixée. Ces ratios continueront à être réduits par la suite, afin de faire baisser les taux d'intérêt pour favoriser les investissements. L'application des ratios prudentiels par les banques sera échelonnée d'ici à la fin de 1996. Elle prévoit notamment qu'à partir du début de 1994, les prêts d'une banque en une devise donnée ne pourront excéder $10 \%$ de son capital et que l'ensemble de ses prêts en devises représenteront au maximum $20 \%$ de son capital. D'autre part, après la fin de 1996, l'encours des prêts à un client donné ne pourra pas dépasser $30 \%$ du capital d'une banque.

Le fonds de garantie des dépôts instauré par les amendements à la loi bancaire de 1957 adoptés les 4 juin 1992 et 11 mars 1993, doit être mis en place à la même date. Une évaluation des créances des banques est également prévue. Les banques seront par ailleurs autorisées, en décembre 1993, à fixer elles-mêmes la tarification de leurs services. Une loi supprimant les planchers et plafonds des rémunérations et tarifs des banques sera présentée au Parlement avant la fin de 1994.

En matière de privatisation, toutes les participations étatiques dans les banques mixtes doivent avoir été cédées d'ici à la mi-1995, selon le calendrier suivant : $20 \%$ des parts de l'Etat doivent avoir été vendues à la mi-1994, 40 \% supplémentaires à la fin de 1994 et les $40 \%$ restants au semestre suivant. Ce projet, qui bénéficie d'un financement de la Banque mondiale, vise 23 établissements, dont 11 joint-ventures égypto-étrangères. De plus, une des quatre grandes banques entièrement publiques (Banque du Caire, Banque d'Alexandrie, National Bank of Egypt ou Banque Misr) sera privatisée avant la fin de 1995. Le gouvernement égyptien précise cependant qu'il «ne garantit la réalisation d'aucune vente d'actif public, mais seulement la mise en œuvre des mesures arrêtées avec la Banque mondiale pour la réalisation des ventes». Il n'a donc pris aucun engagement de résultat

Le projet de loi sur les assurances, qui doit être soumis au Parlement avant la fin de 1993, alignera les conditions égyptiennes sur les normes internationales de fonctionnement de ce secteur. L'application de la nouvelle toi sera accompagnée du renforcement du contrôle de l'Egyptian Insurance Supervisory Authority sur la solvabilité des compagnies d'assurances. A la fin de 1994, un nouveau texte sera soumis au Parlement, afin d'autoriser les investissements étrangers dans ce secteur et de 
libérer la fixation des primes. La libéralisation des assurances prévoit par ailleurs la privatisation des sociétés mixtes du secteur avant la fin de 1994 et de deux compagnies publiques, dont la première avant la fin de 1995. Comme pour les banques, le gouvernement ne s'engage toutefois pas formellement à réaliser ces ventes.

L'environnement législatif du marché financier sera amélioré avant la fin de 1994 afin d'assurer la transparence nécessaire aux transactions et de les faciliter. Un système d'information sur les sociétés cotées sera mis en place et l'informatisation des transactions sera engagée dans un deuxième temps. Un fonds de protection des investisseurs sera institué à la mi-1994, date à laquelle les dispositions de la nouvelle loi du 22 juin 1992 sur le marché financier et la réforme de la Capital Markets Authority, qui supervise l'activité boursière, doivent avoir été mises en vigueur. Des réformes du système des retraites et de la sécurité sociale sont enfin prévues de manière progressive à partir de 1994, afin d'aligner leur mode de fonctionnement sur les standards internationaux.

Restructuration du secteur public et privatisations

La réforme des entreprises publiques visera à restreindre la taille du secteur public et à améliorer sa rentabilité. L'investissement public devrait ainsi pouvoir se stabiliser à environ 7,5\% du PIB et être dirigé vers l'éducation, la santé, l'enseignement et les infrastructures. Parmi les 313 entreprises publiques relevant de la loi 203 de 1991 sur le secteur public, le Bureau des entreprises publiques en a identifié 75 dont l'endettement nécessite une restructuration financière et 51 dont les pertes justifient une recapitalisation ou la liquidation. Les dépenses de recapitalisation ou de restructuration financière seront financées par le produit de la vente des 125 entreprises considérées comme privatisables. D'autre part, le gouvernement n'apportera plus sa garantie aux emprunts, y compris extérieurs, des entreprises publiques, qui seront soumises au droit commun des affaires. Chaque exercice budgétaire comprendra des allocations pour les sociétés à privatiser ou à restructurer. Pour les premières, elles financeront recapitalisation et licenciements, et pour les secondes, elles seront limitées aux sommes que les entreprises concernées n'auront pas pu lever en bourse. Les sociétés non viables seront liquidées, selon un calendrier mis au point en accord avec les institutions financières internationales.

Un échéancier est arrêté pour les privatisations jusqu'à la mi-1995. En 1991 au contraire, seuls des engagements vagues avaient été pris, ce qui a contribué aux atermoiements de l'Égypte dans ce domaine. Selon la Banque mondiale, le produit des privatisations engagées depuis 1990 s'élevait à 1,17 milliard LE à la mi-1992, mais, hormis les entreprises locales, cette somme ne provient que de cessions d'actifs publics et non de privatisations d'entreprises. Le calendrier mis au point distingue trois contingents de sociétés privatisables, correspondant à trois programmes de privatisation pour 1993-94, 1994-95 et 1995-96. Ces années fiscales sont celles au cours desquelles ces trois lots respectifs doivent commencer à être cédés, mais n'avoir pas entièrement été vendus. En effet, le calendrier des privatisations retient des objectifs semestriels de cessions d'actifs, calculés en pourcentage de la valeur comptable " historique " (book values at historical cost) de l'ensemble des sociétés relevant de la loi 203, telle qu'estimée par le Bureau des entreprises publiques à la fin juin 1991, soit 73 milliards LE. Durant les deux premières années de l'accord avec le FMI, 25 \% de ce montant doivent avoir été cédés, soit une somme théorique de 18,6 milliards LE, mais qui peut s'avérer autre si le produit effectif des ventes des actifs publics diverge de 
l'estimation de leur valeur à lin juin 1991. Ceci est d'autant plus probable que l'estimation de cette valeur par des sources indépendantes n'avait pas commencé à cette date.

Aucune date limite n'est par contre fixée pour la cession des 22,5\% restants (soit 16,1 milliards LE) des actifs dont la privatisation est prévue par les programmes 1994-95 et 1995-96, qui porteraient le montant global des cessions à $47,5 \%$ des actifs privatisables. Le gouvernement égyptien ne s'engage en fait à mener à terme avant l'échéance de l'accord avec le FMI que le programme de privatisation 1993-94, laissant pour un avenir imprécis $40 \%$ des cessions prévues par le programme 1994-95 et 80\% de celles inscrites au programme 1995-96, soit au total près de la moitié (46,3\%) des actifs concernés par les programmes de privatisation. Les engagements de cessions d'actifs vont en s'accroissant, de 910 millions LE au deuxième semestre 1993 à 10 milliards LE au premier semestre 1995, comme l'indique le tableau suivant :

Calendrier des privatisations (engagements de cession effective en millions LE)

\begin{tabular}{|c|c|c|c|c|c|c|}
\hline & $2^{\mathrm{e}}$ sem. 1993 & $1^{\mathrm{er}}$ sem. 1994 & $2^{\mathrm{e}}$ sem. 1994 & $1^{\mathrm{er}}$ sem. 1995 & Ultérieur & Total \\
\hline $1^{\mathrm{er}}$ contingt & 910 & 2.730 & 2.730 & 2,730 & - & 9.100 \\
\hline $2^{\mathrm{e}}$ contingt & - & - & 2.190 & 4.380 & 4.380 & 10.950 \\
\hline $3^{e}$ conlingt & - & - & - & 2.920 & 11.680 & 14.600 \\
\hline Total & 910 & 2.730 & 4.920 & 10.030 & 16.060 & 34.650 \\
\hline$\%$ cumulé & $5 \%$ & $5 \%$ & $7 \%$ & $25 \%$ & - & $47,5 \%$ \\
\hline
\end{tabular}

* Pourcentage de la valeur comptable totale des entreprises relevant de la loi 203, calculée par le Bureau des entreprises publiques. Les contingents représentent respectivement $12.5 \%, 15 \%$ et $20 \%$ de cette valeur, soit $47,5 \%$ au total, l'engagement pour le troisième contingent portant exactement sur «20\% au moins » de cette valeur.

Source : Tableau construit à partir des données figurant dans le Memorandum of Economic Policies of the Egyptian Govemment, 18 août 1993.

\section{Promotion du secteur privé}

Les investissements à forte intensité énergétique seront retranchés de la «liste négative » des domaines interdits aux investisseurs étrangers à la fin de 1993. Après Ies aménagements apportés en juillet 1993 (voir chronique économique du troisième trimestre 1993, chapitre "économie »), cette liste ne comprendra ainsi plus que les investissements dans les industries militaires et annexes, le tabac et produits dérivés et dans le Sinaï. Les agréments des gouvernorats en matière d'investissement seront également supprimés à la fin de 1993, ainsi que la délivrance de licences par la General Organisation for Industrialisation (GOFI). A la fin de 1994, les procédures d'enregistrement des nouvelles sociétés seront simplifiées. Une réforme de la fiscalité des investissements est prévue à partir de la fin de 1994 afin de limiter les exonérations, de réorienter les zones franches vers les activités exportatrices et de refondre le "statut franc de Port-Saïd. Un projet de loi à cet effet sera soumis au Parlement à la mi-1994. 

publiques et privées doit être soumise au Parlement d'ici la fin juin 1994. Elle établira les règles en matière de faillites, de fusions, de monopole et d'arbitrage. Une politique de libéralisation du marché du travail devant renforcer la mobilité de la main-d'œuvre sera enfin mise en œuvre à partir de 1995, sur les bases d'une étude à réaliser en coopération avec l'Organisation internationale du travail. Toujours dans ce domaine, la réforme du statut de la fonction publique sera engagée à la fin de 1994, suite à une étude que financera à cette fin la Banque mondiale.

Politique tarifaire

La libération des prix se poursuivra comme suit :

- dans le secteur-agricole, les prix et la culture du coton seront totalement libérés à la fin 1993, le gouvernement continuant cependant à réglementer la rotation géographique des différentes variétés cultivées ; la bourse du coton commencera ses activités en septembre 1994 ; à la mi-1995, la dernière culture à être libéralisée sera la canne à sucre ;

- à la suite des augmentations intervenues en juillet 1993 (voir chronique économique du troisième trimestre 1993, chapitre " électricité »), les prix de l'énergie continueront à être relevés, suivant les engagements pris en 1991 : les prix des produits pétroliers et gaziers rejoindront les niveaux internationaux (moyenne annuelle des prix fob à la sortie des raffineries italiennes) à la mi-1995 au plus tard et les tarifs de l'électricité atteindront les coûts de production marginaux à long terme à la même date ;

- dans l'industrie, le gouvernement supprimera les derniers contrôles sur les produits industriels du groupe cinq (hautement subventionnés ou de monopole) à la fin de 1993;

- une loi libérant les loyers des logements neufs et vacants sera présentée au Parlement à la fin de 1993, pour entrer en vigueur l'année suivante. Une deuxième loi libérant l'ensemble des loyers et éliminant les subventions à l'habitat sera soumise au Parlement un an plus tard, dont l'application sera progressive à partir de 1995 ;

- le programme de relèvement des tarifs des transports ferroviaires sera enfin poursuivi, comme convenu en 1991, pour couvrir les prix de revient en 1997-98.

Libéralisation du commerce extérieur

Outre les mesures adoptées en juillet 1993 (voir chronique économique du troisième trimestre 1993, chapitre « économie »), le calendrier de la libéralisation du commerce extérieur prévoit :

- pour la fin de 1993, une réduction du plafond des droits de douane à $70 \%$ et une baisse de 10 $\%$ des droits frappant les articles actuellement taxés entre $30 \%$ et $70 \%$. Les droits supportés par une série de biens d'équipement seront par ailleurs fixés à $5 \%$ ou $10 \%$, les taux sur les autres devant être abaissés au même niveau avant la fin 1995, sauf rares exceptions. Des projets de loi autorisant les investissements étrangers dans le commerce, d'une part, l'importation (sous réglementation sanitaire) et l'exportation de coton, d'autre part, seront soumis au Parlement. Une nouvelle loi régissant le contrôle de la qualité, les normes, la concurrence déloyale et les mesures anti-dumping entrera en vigueur, dans le cadre des règles du GATT et sans que des obstacles non-tarifaires puissent être renforcés ou institués. La nomenclature douanière internationale harmonisée sera enfin introduite ;

-à la mi-1994, les cigarettes et les voitures de cylindrée supérieure à $1.600 \mathrm{~cm} 3$ intégreront la grille générale. Un taux exceptionnel de $100 \%$ demeurera provisoirement pour les voitures de 1.300 à $1.600 \mathrm{~cm} 3$, qui sera par la suite abaissé à hauteur du taux maximal. Les dérogations pour le secteur touristique seront supprimées, exception faite des alcools. L'interdiction d'importer les volailles sera levée, le sort des textiles dépendant quant à lui du

Égypte/Monde arabe, 15-16 | 1993 
renouvellement en cours de l'accord international « multi-fibres ». Des mesures contre le dumping et la concurrence déloyale et pour le respect des normes entreront en vigueur. L'interdiction d'exporter des peaux brutes sera supprimée. La réforme en cours de la procédure douanière sera enfin achevée ;

- à la fin de 1994, un nouvel abaissement du plafond des droits de douane à 60 \% et une nouvelle baisse de $10 \%$ des droits frappant les articles auparavant taxés entre $30 \%$ et $60 \%$ entreront en vigueur ;

- à la mi-1995, l'interdiction d'exporter les ferrailles sera levée ;

- à la fin de 1995 enfin, interviendront l'abaissement du plafond des droits de douane à 50 \% et une baisse supplémentaire de $10 \%$ des droits frappant les articles auparavant taxés entre 30 $\%$ et $50 \%$. Une nouvelle réduction, à un taux non encore arrêté, entrera en vigueur à la fin de 1996.

Les perspectives macro-économiques

Pour l'année fiscale 1993-94, les prévisions gouvernementales sont les suivantes :

- croissance réelle du PIB de $2 \%$, contre 0,3 \% en 1991-92 et 0,5 \% en 1992-93;

- baisse de l'inflation de 11,1\% en 1992-93 (contre un objectif de 10\% fixé par le programme d'ajustement structurel) à 9,5\%, mais $21,1 \%$ en 1991-92. Ce résultat devrait provenir de la poursuite de la politique déflationniste du programme d'ajustement, d'un faible relèvement des tarifs publics ou administrés et du maintien d'un cours élevé de la livre, qui permet de modérer l'inflation importée ;

- diminution de l'excédent de la balance globale de 3,9 milliards \$ en 1992-93 à 2 milliards \$, en raison de la hausse des importations et des baisses des recettes touristiques et des transferts des émigrés ;

- réduction du déficit budgétaire de l'équivalent de 4,7\% du PIB en 1992-93 à 2,6 \% du PIB, hors recettes de privatisation, qui seront affectées à la restructuration du secteur public (non budgétisée). Ce déficit est nettement inférieur aux 6,5\% que prévoyait la loi de finances votée quelques jours avant ces engagements ${ }^{10}$, ce qui ridiculise le rôle des députés dans un domaine aussi important de la vie du pays. Pour 1992-93, le gouvernement a débudgétisé le financement des entreprises publiques relevant de la loi 203, ce qui, ajouté à une baisse plus importante qu'escomptée des intérêts dus sur la dette extérieure, devrait ramener le déficit budgétaire des 6,5\% initialement prévus à 3,5\% du PIB, selon les prévisions gouvernementales, mais à $4,7 \%$ selon le FMI. A noter que les dépenses additionnelles occasionnées au budget par le tremblement de terre du 12 octobre 1992 ont été couvertes par l'aide étrangère prodiguée à sa suite ${ }^{11}$. Puisque cette dernière ne figure pas en recettes et que ces dépenses ont représenté $0,6 \%$ du PIB, le déficit budgétaire réel revient à $4,1 \%$ du PIB en 1992-93, contre un objectif de 3,5\% fixé par le programme d'ajustement ;

- la réduction requise du déficit budgétaire engage à un net surcroît d'austérité, les recettes devant augmenter de un point de PIB et les dépenses diminuer de 1,1 point. Les subventions aux produits de première nécessité seront réduites de 525 millions LE, en relevant notamment les prix des huiles végétales, et leur gestion sera améliorée de façon à limiter le déficit de la General Authority for Supply of Commodities de 2,9 milliards LE en 1992-93 à moins de 2 milliards LE en 1993-94. Les subventions aux engrais et pesticides seront définitivement supprimées, ce qui économisera 176 millions LE, et 75 millions LE de dépenses budgétaires supplémentaires seront épargnées grâce à la disparition des subventions aux prêts aux exploitants agricoles individuels. L'augmentation des salaires sera limitée à $15 \%$ en 1993-94, contre 20 \% l'année précédente. L'encours des crédits au secteur public sera réduit de 1,6 milliard LE en 1993-94, les dépenses d'investissement étant limitées à 10,5 milliards LE, soit 7,2\% du PIB, contre 7,7\% en 1992-93. Les investissements 
prévus au plan quinquennal 1992-93/1996-97 seront revus à cet effet. Les déboursements du Fonds social, qui seront budgétisés, atteindront 440 millions LE. Des plafonds trimestriels ont été fixés pour les emprunts intérieurs nets du gouvernement, des collectivités locales et des établissements publics. 30,27 milliards \$ en 1991, soit 100 milliards LE, alors que le plan 1993-94 indique un chiffre de 134,3 milliards LE pour 1992-93 (contre 118,2 milliards LE estimés pour 1991-92) et que des estimations gouvernementales vont jusqu'à 145,8 milliards LE en 1993-94. Ces estimations divergentes sont à l'origine de la brusque augmentation du PIB par habitant, qui serait passé, selon les chiffres communiqués par l'Égypte au FMI, de 610 \$ en 1991-92 à 740 \$ en 1992-93, ce qui voudrait dire que l'ajustement structurel se serait accompagné d'une hausse record de $21,3 \%$ du niveau de vie en un an. Sa croissance réelle aurait, selon elles, atteint $1,1 \%$ en $1991-92$ et $4,4 \%$ en 1992-93, chiffres contredisant totalement ceux du FMI, cités plus haut. Or la Banque mondiale estimait le revenu par tête à 620 \$ en 1991. Ces contradictions constituant une règle plutôt qu'une exception dans la statistique économique égyptienne, les autorités se sont engagées à publier des statistiques améliorées sur la production intérieure brute à partir de la mi-1994 et sur l'emploi, les salaires, les prix, la production industrielle et la balance des paiements dès la fin de 1993.

La deuxième phase de l'ajustement sera délicate

Pour apprécier les divers éléments de cette nouvelle phase de l'ajustement structurel, il convient d'abord d'examiner dans quelles conditions elle succède à la première. Les principales interrogations qui l'entourent ont trait aux privatisations. Les chances de réussite de ce programme ne dépendent enfin pas uniquement de son contenu, mais de l'environnement dans lequel il sera appliqué.

39

Une remarque préalable à l'analyse est utile : les programmes d'ajustement structurel dans le tiers-monde ont hérité, des politiques économiques suivies auparavant, un certain goût du secret. Aux susceptibilités qui entouraient traditionnellement, durant la guerre froide, toute donnée économique nationale, est venue s'ajouter la confidentialité des rapports avec les institutions financières internationales. Par ailleurs, plus ces dernières se sont engagées dans la gestion des économies sinistrées qu'elles devaient remettre à flot, plus elles ont elles-mêmes acquis la manie de la 
dissimulation, officiellement par égard envers les gouvernements locaux, en pratique pour se préserver aussi des critiques vis-à-vis d'échecs éventuels. Il existe une gradation dans le secret entre la Banque mondiale et le FMI, plus puissant. Cette situation n'est pas pour rien dans les attaques récurrentes et souvent infondées - par indisponibilité de fondements autant que par préjugés idéologiques - dont ces institutions sont l'objet, particulièrement en Égypte. Les commentateurs les mieux informés en sont donc quelquefois réduits aux interprétations, si ce n'est aux hypothèses, en ce qui concerne notamment les relations entre les interventions du FMI et de la Banque mondiale dans un pays sous ajustement, qui ont elles aussi leur importance et leur complexité. Il est probablement superflu de préciser que plus un régime doute de sa stabilité, plus reste opaque la politique d'ajustement structurel qu'il met en œuvre. Par contre, il peut être utile d'ajouter que cet état de fait constitue un important obstacle au succès du programme d'ajustement structurel, puisque seule une transparence de bon aloi permet aux acteurs économiques d'optimiser leur comportement.

De la première à la deuxième phase de l'ajustement

40 A l'issue de l'accord de confirmation, il apparaît que l'Égypte a obtenu la deuxième tranche de réduction de sa dette sans avoir rempli toutes les conditions initialement requises à cet effet, la politique de stabilisation ayant été mise en œuvre, mais les mesures d'ajustement structurel beaucoup moins. Aucune rigueur ne lui ayant été tenue de son retard, il est probable que ce précédent influencera son comportement futur.

41 La comparaison des promesses initiales et des nouveaux engagements montre en effet que le gouvernement égyptien a réussi à obtenir du FMI un rythme de réformes plus lent que demandé, en lui opposant de manière récurrente l'argument du danger social qu'aurait représenté selon lui une mise en œuvre plus rapide. Dès le premier examen de l'accord de confirmation, un retard était apparu en matière de contrôle monétaire. Bien que conduit avec six mois de délai sur le calendrier initial, la deuxième évaluation soulignait les retards en matière d'ajustement structurel. La réforme de la fiscalité des revenus devait être adoptée en 1992. Les salaires du secteur public ont été augmentés de $22 \%$ en juillet 1992, soit une hausse réelle de $12 \%$, contre une baisse de $15 \%$ prévue, et le dérapage du projet de budget 1992-93 a mis plusieurs mois à être corrigé. La réforme de la fonction publique devait également être engagée dans le cadre de l'accord de confirmation. La loi unifiant les conditions de l'activité économique, publique et privée, devait être soumise au Parlement à la fin de 1992. Enfin, les activités du Fonds social ont été bien moindres qu'escompté, surtout parce que les réductions d'effectifs envisagées dans les entreprises publiques ne sont pas encore intervenues. 40.000 licenciements par semestre étaient initialement prévus à partir du début de 1992.

42 L'Égypte a également accusé un retard de plus d'un an dans la conclusion de l'accord d'application des décisions du Club de Paris avec l'Italie et dans le règlement de 767 millions \$ d'arriérés sur la dette contractée à l'égard des pays non-membres du Club de Paris. D'autre part, bien que les engagements pris en matière de privatisation aient dès l'origine manqué de précision ${ }^{12}$, il est clair que leur mise en œuvre accuse un retard important. La liste des 45 premières sociétés privatisables ${ }^{13}$ avait notamment été arrêtée dès le premier trimestre 1992, pour que leur vente puisse être achevée avant 
juillet 1993. Le problème des privatisations, politiquement le plus délicat pour le régime, pourrait s'envenimer à l'avenir.

Le problème des privatisations

43 En effet, si le FMI a très précisément balisé la voie à suivre en matière douanière et fiscale, il n'en va pas de même dans le domaine des privatisations. Une analyse attentive montre en effet que les engagements pris à ce sujet dans la lettre d'intention ne sont pas aussi rigoureux qu'ils en ont l'air.

Le texte de la lettre d'intention adressée au Fonds est tout d'abord ambigu en plusieurs points. Les promesses de cessions d'actifs publics sont prises uniquement en pourcentage de la valeur comptable globale des entreprises relevant de la loi 203, le gouvernement ne s'étant pas engagé sur un nombre de sociétés à privatiser et n'ayant pas non plus mentionné la liste des sociétés privatisables. Il est vrai que le FMI laissera le contrôle de cette partie de l'ajustement structurel à la Banque mondiale, avec laquelle l'Égypte n'a pas encore renouvelé son accord, si bien qu'on peut imaginer que la Banque demandera des engagements plus précis que le Fonds. Obtiendra-t-elle satisfaction, alors qu'elle n'a aucun moyen de pression sur le gouvernement égyptien ? Le mode de calcul retenu laisse par ailleurs le champ à des révisions : si les résultats des banques ou cabinets auxquels sont confiées les évaluations des entreprises privatisables divergent trop de leur valeur de base estimée ou bien s'ils indiquent des montants inattendus pour les restructurations préalables, les autorités égyptiennes ne serontelles pas tentées de le remettre en cause?

D'autre part, le gouvernement ne promet dans sa lettre d'intention aucune réalisation de vente, employant trois fois la même formule à propos des privatisations des entreprises publiques, des banques et des sociétés d'assurances. Cette clause de style montre que le gouvernement ne s'engage qu'à faire évaluer les entreprises privatisables - grâce à des financements internationaux - et à lancer des procédures de vente, sans préjudice de leur caractère effectif ${ }^{14}$. Les appels d'offres lancés au premier semestre 1993 pour le premier lot de sociétés privatisables sont symptomatiques à cet égard : leurs dates de clôture ont été fréquemment repoussées, puis surtout ils ne préjugent en rien de cessions effectives, qui peuvent être indéfiniment reportées si les autorités considèrent insuffisantes les offres reçues. Seules deux sociétés importantes ont été privatisées en 1993 à la suite de ces appels d'offres, el-Nasr Bottling Co. (Coca Cola) et l'Egyptian Bottling Co. (Pepsi Cola).

On a par ailleurs remarqué que le gouvernement ne s'est engagé à céder que 53,7\% (18,6 milliards LE sur un total de 34,65 milliards LE) des actifs inclus dans les programmes de privatisations. De plus, ces derniers ne représentent que $47,5 \%$ des actifs privatisables (73 milliards LE), selon le mode de calcul adopté. Si l'on retient enfin l'estimation gouvernementale de la valeur des entreprises publiques non privatisables, soit 130 milliards LE, il apparaît que l'État égyptien ne s'est pour le moment engagé à privatiser que $9,2 \%$ des actifs qu'il détient dans les entreprises entièrement publiques, plus une part importante, mais minoritaire, de ses participations dans le secteur financier. D'autre part, le sort réservé à ses participations dans le secteur dit d'investissement (relevant des lois 43 de 1974 et 230 de 1989) régissant les investissements étrangers) reste obscur. En effet, la nature des opérations de privatisations n'est pas mentionnée clairement dans la lettre d'intention: les engagements pris concernent-ils les entreprises relevant de la loi 203, comme implicite, ou le produit des ventes de participations étatiques dans le secteur mixte entrera-t-il en compte? Le terme de 
«privatisation » employé par le gouvernement est-il entendu comme la vente d'actifs étatiques au secteur privé, de participations de l'État au capital, minoritaires ou majoritaires, ou même de sociétés entières?

Pour analyser ce dernier trait, il est intéressant d'examiner le seul document officiel traitant des privatisations publié jusqu'à présent, tout en notant qu'il n'a aucun caractère juridique, nul décret ou loi n'ayant jusqu'à présent abordé ce domaine. Avant que la Banque mondiale ne débloque la deuxième tranche de son prêt d'ajustement structurel, le Bureau des entreprises publiques avait en effet publié le 14 février 1993 une brochure intitulée Procédures et lignes directrices du programme gouvernemental d'élargissement du mode de propriété, de restructuration, d'incitations des employés et du secteur public. Ce titre délaisse significativement le mot "privatisation" (takhsis ou khaskhasa en arabe) pour la formule : «élargissement du mode de propriété ». Dans sa version anglaise, le titre arabe est pourtant traduit par «programme gouvernemental de privatisation ", mais il est immédiatement précisé « qu'en cas de discordance entre les textes anglais et arabe, la version arabe prévaudra ». La nécessaire exégèse de ce texte répond-elle à la même fin que celle de la loi $203^{15}$, à savoir ménager des susceptibilités, ou se réserver une issue de repli ?

Une opération de privatisation y est définie (p. 12) comme "le transfert au secteur privé des principaux actifs productifs d'une entreprise publique ou de toutes les actions détenues par l'État dans une joint-venture relevant des lois 43 ou 230. Pourquoi ne pas avoir utilisé le critère traditionnel de la répartition du capital ? Par souci de clarté, le texte précise que "les entités du "secteur privé » dans ce contexte excluraient toute entité détenue ou contrôlée par le secteur public » et indique (p. 6) que «la vente d'une entité publique à une autre entité publique ne sera pas considérée comme une privatisation».

49 On peut enfin remarquer qu'aucune stratégie n'est définie dans la lettre d'intention en matière de privatisation. Le fait que leur produit ne doive pas être affecté à la réduction - draconienne - du déficit budgétaire prévue par ailleurs, alors que cela pourrait être l'un des moyens privilégiés à cette fin, suivant l'expérience d'autres pays, est étonnant. Le FMI aurait-il si peu confiance dans la bonne marche des privatisations qu'il préfère prévoir d'autres moyens de parvenir à son objectif fiscal prioritaire ? Laissé à la Banque mondiale, dont l'importance est seconde, par rapport au Fonds, ce domaine n'est guère éclairci par rapport aux engagements très généraux pris en la matière dans le cadre de la première phase de l'ajustement structurel. Contrairement au document accompagnant le prêt d'ajustement structurel de la Banque mondiale, aucun chiffre n'est avancé cette fois-ci en matière de réduction d'effectifs des entreprises publiques. Il est vrai qu'après d'autres officiels, le nouveau ministre du secteur public, qui est en charge du programme de privatisations, a réaffirmé qu'aucun travailleur du secteur public ne serait licencié à la suite de sa restructuration ${ }^{16}$, déclaration que l'on peut interpréter soit comme soucieuse d'apaiser l'opinion, soit comme révélatrice des intentions gouvernementales réelles, parfaitement dissuasives pour les investisseurs.

Une indication supplémentaire de la dérive enregistrée par rapport à ce qu'escomptait la Banque mondiale en 1991 est l'annonce en octobre dernier, par le directeur général du Fonds social, de l'extension des activités de son organisme aux problèmes de réduction de la pauvreté et de l'environnement, deux des plus récents domaines de prédilection de la Banque mondiale. Les ressources que le Fonds social n'arrive pas à affecter aux licenciés du secteur public sont donc apparemment appelées à une 
utilisation différente de leur destination d'origine. Il semble que le FMI n'ait pas demandé à l'Égypte un programme détaillé de privatisations. C'est ainsi que, dans sa lettre d'intention, le gouvernement s'engage en effet à vendre avant la fin de 1993 les 22 sociétés (dont 6 entièrement publiques) ou actifs de sociétés annoncés comme privatisables en mars 1993 « ou des actifs équivalents d'une valeur comptable (à fin juin 1991) de 1,4 milliard LE». Ce chiffre est supérieur au montant (910 millions LE) de « l'engagement semestriel » mentionné dans le tableau détaillé des mesures à adopter, figurant quelques pages plus loin.

51 Une autre phrase de la lettre d'intention indique la disposition du Fonds, qui a pourtant amendé certains articles de la lettre d'intention avant de l'accepter, à ne pas précipiter les privatisations: dans un paragraphe traitant de la politique monétaire, il est mentionné que cette dernière "permettra aux crédits du secteur privé (y compris les entreprises du secteur public) d'augmenter de plus de 16\% en 1993-94». Il semble en fait qu'à la suite de leur débudgétisation, les investissements des entreprises publiques soient mis sur le même plan que ceux des firmes privées. Dans le même ordre d'idées, le ministre du secteur public, M. Atef Obeid, a affirmé qu'à l'issue des privatisations, une part importante des investissements restera dévolue au secteur public, ce qui impliquera une privatisation de type spécial devant concerner un grand nombre d'entreprises, la privatisation de la gestion $»^{17}$. Les entreprises publiques ne se réduisant pas aux hôtels publics, dont la gestion a été confiée à des sociétés privées, on peut interpréter cette formule comme « une gestion semblable à celle d'une entreprise privée ». Ainsi l'unification prévue des lois régissant le fonctionnement des entreprises, publiques et privées, serait-elle assimilée à un mode de privatisation, ce qui, même accompagné de la poursuite du lent mouvement de privatisations effectives engagé en 1991, jetterait une lumière nouvelle sur les engagements pris vis-à-vis du FMI...

On pourrait multiplier les exemples d'interférences entre secteurs privé et public. C'est ainsi que M. Youssef Boutros-Ghali, réputé le plus libéral des membres du gouvernement, intitulait un article écrit l'été dernier: «Les réformes du marché : à travers une plus forte intervention du gouvernement $»^{18}$. En paraphrasant le " socialisme de marché » dont se réclame depuis peu la Chine, on pourrait qualifier de « dirigisme de marché » la doctrine des dirigeants égyptiens.

Le contexte de la libéralisation

53 L'économie égyptienne pourrait ne pas bénéficier, pour la deuxième étape de son ajustement, du soutien international exceptionnel qui a grandement contribué au succès de la première. Pourtant, la reconduction, en octobre 1993, de M. Hosni Moubarak à la présidence de la République et de M. Atef Sedki à la présidence du Conseil indique que la conduite de la politique économique égyptienne ne sera pas modifiée.

Contrairement aux attentes, le nouveau gouvernement alors formé n'apporte que des changements mineurs, à l'image du remaniement ministériel intervenu au lendemain de la signature de l'accord de 1991 avec le FMI, qui avait déjoué les espoirs des partisans d'une libéralisation accélérée. Bien que nombre de portefeuilles économiques aient changé de mains (économie, industrie, tourisme, travaux publics, habitat, travail, coopération internationale), l'absence d'une nouvelle orientation confirme le fait que les ministres ont une fonction d'exécution et non d'élaboration de la politique égyptienne, domaine réservé au président du Conseil et accessoirement aux vice- 
premier ministres (MM. Wali et Ganzouri), les décisions étant, bien entendu, prises par le président.

Ce remaniement conforte l'ascension de M. Youssef Boutros-Ghali, contrôlé cette fois par la mise de son département sous tutelle de la présidence du Conseil. Le principal changement intervenu le 13 octobre, simultanément au nouveau gouvernement, pourrait en fin de compte être le remplacement de M. Salah Hamed à la présidence de la Banque centrale par M. Ismail Hassan Mohamed, qui présidait la Banque d'Alexandrie (publique). La Banque centrale abrite le bureau du représentant résident du FMI en Égypte. La politique officielle de promotion du secteur privé ne se traduit pas par l'entrée au gouvernement de personnalités issues de ses rangs, dont la déception doit être à la mesure des efforts qu'elles ont prodigués à cette fin. Or, le succès de la deuxième phase de l'ajustement structurel dépend en grande partie de son accueil par le secteur privé, dont les investissements doivent relayer ceux de l'État. Le manque de confiance des hommes d'affaires envers les gestionnaires de l'économie crée un climat peu propice à une telle évolution. On peut interpréter dans le même sens l'arrivée cet été, à la tête de la Fédération des industries égyptiennes, de M. Mohamed Farid Khamis, l'un des patrons égyptiens les plus dynamiques et les plus puissants, connu pour être proche du président Moubarak, comme un indice supplémentaire de la faiblesse politique persistante du secteur privé égyptien. Après avoir conféré une large audience à l'Association des hommes d'affaires du Dix-de-Ramadan, en marge des canaux bureaucratiques, le fait qu'il prenne la présidence de cette institution intégrée de longue date à la bureaucratie économique égyptienne est significative, au choix, du chemin que le secteur privé doit continuer à emprunter pour faire valoir ses intérêts, de celui qui lui reste à parcourir à cette fin ou même du dévoiement des intérêts catégoriels des entrepreneurs égyptiens par un système bureaucratique qui ne reconnaît que ceux qui se plient à ses structures. A l'heure où d'autres pays de la région (Maroc, Tunisie...) sortent de l'ajustement, aucune accélération des réformes ne semble d'actualité en Égypte.

Le principal obstacle au succès du programme égyptien d'ajustement structurel ne paraît pas tant être la volonté de l'État de le mettre en œuvre - il aurait pu refuser de reconduire son accord avec le FMI - que la situation dans laquelle il agit. L'application des nouvelles lois pourrait se heurter à l'absence quasi-totale de mobilisation des Égyptiens en faveur des réformes. A l'inverse des situations prévalant en Europe de l'Est ou en Chine, il n'existe pas en Égypte de mouvement populaire vers la libéralisation de l'économie, comme il y en eut pour son étatisation. C'est pourquoi un vaste mouvement de privatisations semble difficile à accomplir dans les conditions actuelles.

En conséquence, le pouvoir n'a pas davantage qu'en 1991 exposé publiquement les nouvelles mesures d'ajustement économique, la diffusion des engagements décrits cidessus ayant été la plus restreinte possible. Cette situation alimente tout au plus des rumeurs, généralement démenties par les responsables. Les informations distillées de temps à autre sont agrémentées de propos lénifiants de responsables promettant la sauvegarde des acquis sociaux tout en proclamant le caractère inéluctable de l'austérité. L'économie est plus que jamais soustraite au débat et sa libéralisation est perçue par la plupart moins comme un virage que comme la poursuite de l'itinéraire suivi depuis l'infitah.

Conclusion 

pression sur les pays ayant signé des accords d'ajustement structurel, le déblocage des tranches des prêts qu'ils leur consentent pour accompagner ces accords. Les difficultés financières de ces pays sont en général telles que ces déblocages leur sont indispensables. Le cas atypique d'une Égypte n'ayant jamais connu une aisance financière telle que depuis le début de l'ajustement de son économie, rend inopérant ce type traditionnel de "conditionnalités ", remplacé ici par les étapes de la réduction de sa dette extérieure. Le nouvel accord avec le FMI a été conclu pour une période de trois ans s'achevant le 30 juin 1996. La dernière tranche de réduction de la dette étant actuellement envisagée pour la fin de 1994, le Fonds ne disposera plus ensuite de ce moyen de pression pour que son programme soit respecté. Si l'Égypte ne remplit pas ses engagements, elle perdra donc 3.28 milliards $\$$ de remises de dettes; si elle les tient, elle gagnera par la suite une grande autonomie de politique économique. L'annonce par le gouvernement égyptien, dès le début de sa lettre d'intention au FMI, qu'il ne touchera pas à l'argent que ce dernier lui propose indique qu'il espère se passer de lui dès que possible.

D'aucuns, parmi le personnel du Fonds, ont dû percevoir la déclaration de ce refus comme superflue ou même présomptueuse. La situation inédite de l'Égypte met cependant en évidence un défaut dans la stratégie du FMI à l'égard des pays en voie d'ajustement économique, qui doit laisser mal à l'aise cette institution peu encline à voir son autorité contestée. Le cas de l'Égypte doit préoccuper encore davantage la Banque mondiale, qui y a pourtant investi lourdement en ouvrant un bureau de représentation. Organisme destiné à l'origine à financer des projets, elle avait déjà ressenti comme contre nature sa dérive vers le financement des programmes d'ajustement structurel, qui l'ont amenée à devenir le complément du FMI. Les conditionnalités de ses prêts la maintenaient cependant dans son rôle bancaire. Or, la Banque mondiale est désormais appelée à faire de la politique économique en Égypte sans liaison avec des prêts, à devenir en quelque sorte conseil en développement sans être rémunérée à cette fin. Le rôle que lui a conféré en 1992 la communauté internationale pour le développement des Territoires occupés est conforme à cette évolution, mais imaginerait-on par exemple la Banque Lazard travailler gratuitement ? Comment pourra-t-elle justifier cette fonction auprès de ses actionnaires? De plus, cette situation la subordonne totalement au FMI pour s'assurer que ses conseils au gouvernement seront bien suivis.

Les considérations développées dans cet article amènent deux remarques. Tout d'abord - ce n'est qu'une confirmation - les autorités égyptiennes n'ont peut-être ni les moyens ni le désir de mettre en œuvre un programme global (radical) - de restructuration de leur économie, auquel ils préfèrent des mesures partielles et progressives. Deuxièmement - ceci est plus nouveau - cette constatation semble également valable pour le Fonds monétaire international. A-t-il baissé les bras devant l'ampleur de la tâche, à la suite d'instructions américaines ou en raison d'incapacités conceptuelles? Il est également possible qu'il s'agisse d'un signe d'une évolution conceptuelle des interventions du FMI, à mettre en relation avec son succès mondial nouvellement acquis. En gagnant l'universalité, sa doctrine s'accommode de distorsions, préservant certes l'essentiel.

61 Un rapport sur la situation économique de l'Afrique subsaharienne achevé en 1993 par la Banque mondiale estime qu'en dépit de ses objectifs, l'ajustement structurel n'y a pas 
entraîné de transfert d'autorité de l'État vers le secteur privé autochtone, mais vers les institutions financières étrangères ${ }^{19}$. A défaut de stratégie nouvelle, ceci dénote une prise de conscience concordant avec nos observations. Ces dernières montrent que cette évolution est subie. Encore accrochées à leurs convictions, les institutions financières internationales poussent en apparence à une privatisation accélérée, de peur probablement qu'une attitude plus conciliante ne ralentisse encore leur tempo. En réalité, elles acceptent des compromis non explicites et fluctuants en fonction des rapports de forces, ce qui met à mal leur réputation de toute-puissance.

Le renouvellement de l'accord entre le FMI et l'Égypte engage cette dernière, dans la deuxième phase d'un ajustement structurel qui adaptera l'infrastructure réglementaire et institutionnelle de l'économie égyptienne aux critères d'une économie de marché. Cette phase laisse donc la part belle aux interventions de l'État, qui doit organiser son retrait de l'économie. La vérité conjoncturelle du paradoxe que soulignait le titre de l'article de M. Boutros-Ghali ne doit pas faire oublier qu'en Égypte comme ailleurs, le fonctionnement d'une économie de marché ne se décrète pas, même sous l'impulsion des institutions financières internationales.

\section{NOTES}

1. Pour une analyse des accords conclus en 1991 par l'Égypte avec les institutions financières internationales, voir notre article « Le programme de stabilisation et d'ajustement structurel de l'économie égyptienne ", in Égypte/Monde Arabe, 9, 1er trimestre 1992, p. 13-46. Pour une recension détaillée des relations préalables de l'Égypte et du FMI, cf. T. Niblock, « The Egyptian Experience in Regional Perspective: International Factors and Economic liberalization in the Arab World », in L. Blin (dir.), L'économie égyptienne. Libéralisation et insertion dans le marché mondial, L'Harmattan, Paris, 1993.

2. Cette décision n'a pas été annoncée publiquement et n'a donc pas reçu de motivation officielle (cf. Égypte/Monde Arabe, 12-13, 4e trimestre 1992-1er trimestre 1993.p.401).

3. Égypte/Monde Arabe, 11, $3^{\mathrm{e}}$ trimestre 1992, p. 250.

4. Fonction équivalente à celle de secrétaire d'État en France. Le 14 octobre suivant, M. Youssef Boutros-Ghâli remplace M. Maurice Makramallah comme ministre d'État à la coopération internationale. Contrairement à ce dernier, il est placé auprès de la présidence du conseil, M. Atef Sedki cumulant la primature avec la fonction de ministre de la coopération internationale. Autre signe de l'importance conférée à M. BoutrosGhâli, il est placé au premier rang des ministres d'État dans le protocole, alors qu'il est le plus jeune membre du gouvernement. Né en 1952, M. Youssef Boutros-Ghali est diplômé de la faculté d'économie et de sciences politiques de l'université du Caire (1974) et titulaire d'un doctorat du Massassuchets Institute of Technology (1980). Après avoir enseigné à l'Université du Caire, il entre en 1981 en tant qu'économiste senior au Fonds monétaire international à Washington. Il participe à ce titre à la préparation des programmes de réforme économique philippin, chinois, brésilien, argentin, mexicain, sénégalais et ivoirien. Il est nommé en 1986 conseiller du Premier ministre pour les 
affaires économiques et cumule cette fonction, à partir de 1988, avec celle de professeur d'économie à l'Université du Caire. En 1991, M. Youssef Boutros-Ghali devient en outre conseiller du gouverneur de la Banque Centrale, puis il fonde et dirige le Centre d'analyses économiques, placé auprès du Premier ministre. Il est également administrateur de la National Bank of Egypt.

5. Al-Ahram. 21 novembre 1993.

6. Égypte/Monde Arabe, 6, $2^{\mathrm{e}}$ trimestre 1991, p. 248.

7. Égypte/Monde Arabe, 12-13, $4^{\mathrm{e}}$ trimestre 1992-1er trimestre 1993. p. 429.

8. Égypte/Monde Arabe, 7, $3^{\mathrm{e}}$ trimestre 1991, p. 235.

9. Égypte/Monde Arabe, 9,1 ${ }^{\mathrm{er}}$ trimestre 1992, p. 13-46.

10. Égypte/Monde Arabe, 14, $2^{\mathrm{e}}$ trimestre 1994.

11. Voir à cet égard Égypte/Monde Arabe, 12-13, p. 399. Les dépenses étatiques de réparation à la suite du tremblement de terre se sont donc élevées à 800 millions LE jusqu'à la fin de juin 1993, soit trois à cinq fois moins que les évaluations des dégâts avancées par les dirigeants égyptiens au moment où affluait l'aide étrangère et 300 millions LE de moins que le montant atteint par cette dernière, selon nos estimations. Quoiqu'il soit probable que de nouvelles dépenses soient imputées sur le budget 1993-94, il apparaît clairement que les charges pour l'Etat ont été surestimées, selon une recette éprouvée lors de la guerre du Golfe. Le tremblement de terre n'a donc pas affecté les finances publiques, contrairement à l'argument présenté à son lendemain pour obtenir un prolongement de raccord avec le FMI.

12. Égypte/Monde Arabe, 9, p. 25.

13. Égypte/Monde Arabe, 14, p. 252.

14. Encore cette affirmation procède-t-elle d'une vision optimiste des choses, si l'on se réfère à l'expérience d'une grande banque européenne, que les autorités égyptiennes ont contactée pour évaluer plusieurs entreprises, puis à laquelle elles ont demandé de réduire de... 70 \% le devis présenté à cet effet, manière détournée de décliner son offre. Tous les moyens semblent bon, y compris s'ils sont cavaliers, pour repousser les échéances, tout en respectant formellement les instructions des institutions financières internationales.

15. Voir notre article cité, in Égypte/Monde Arabe, 9, p. 23.

16. Al-Ahram, 3 novembre 1993.

17. Al-Gumhuriyya, 17 novembre 1993.

18. Introduction au numéro 5 (septembre 1993) de L'Antenne, périodique du Club d'affaires franco-égyptien (Le Caire).

19. Alternatives Economiques, novembre 1993, p. 58.

INDEX

Mots-clés : économie, ajustement structurel, libéralisation, FMI 
AUTEUR

LOUIS BLIN

CEDEJ 